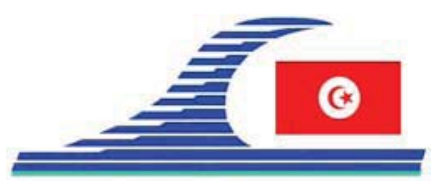

Conférence Méditerranéenne Côtière et Maritime EDITION 1, HAMMAMET, TUNISIE (2009)

Coastal and Maritime Mediterranean Conference

Disponible en ligne - http://www.paralia.fr-Available online

\title{
Evolution des flèches littorales le long d'une côte sableuse microtidale : cas de la flèche sud d'Oued Sourrag (Golfe de Gabès, Tunisie)
}

\author{
Ikram BARDI ${ }^{1,3}$, Mongi SOUAYED ${ }^{2,3}$, Saadi ABDELJAOUED ${ }^{1,3}$
}

1. Département de Géologie, Fac. des Sc. de Tunis, Univ. de Tunis-El-Manar, Tunisie. ikram.bardi@yahoo.fr

2. Département Art et Métiers, Institut Supérieur des Beaux-Arts de Sousse, Tunisie. mongi_souayed@yahoo.fr

3. Laboratoire des Ressources Minérales et Environnement, F.S.Tunis, Tunisie.

\section{Résumé :}

La frange littorale au Sud de la ville de Gabès, est une côte sableuse microtidale marquée par l'individualisation d'une flèche littorale au Sud de l'oued Sourrag, qui a évolué et changé en une dizaine d'années, d'une forme individualisée à une forme rattachée au cordon littoral de l'estran. Ceci est mis en évidence par l'analyse des photos aériennes et satellitaires. L'évolution de cette flèche est contrôlée d'une part, par les apports sédimentaires continentaux qui alimentent la côte lors des crues de l'oued Sourrag; d'autre part, par la dérive littorale NW-SE. Le Système de Modélisation Côtière (S.M.C.), a été utilisé comme outil pour identifier l'hydrodynamisme de la région. Les chenaux de marée contraignent la croissance de la flèche et la maintiennent en équilibre dynamique lorsqu'ils se développent au niveau de son extrémité. L'hydrodynamisme de la côte de Gabès est dominé par les courants littoraux, les courants tidaux étant faibles. Ces courants littoraux jouent un rôle amplificateur dans l'évolution des côtes sableuses.

\section{Mots clés :}

Côte sableuse - Morphologie côtière - Evolution morphologique - Flèche littorale Hydrodynamisme - Dérive littorale - Chenaux de marées - Oued Sourrag - Gabès

\section{Introduction}

Au niveau du Golfe de Gabès (Sud tunisien), la frange littorale entre la ville de Gabès et le village de Kettana se distingue par des plages sableuses (BEN OUEZDOU, 1987 ; MAGHERBI, 1995 ; BARDI, 2002), microtidales et à pente faible. Durant ces dernières années, on a noté l'apparition de flèches littorales abritant des lagunes parcourues de chenaux tidaux. La flèche au Sud d'oued Sourrag est la plus développée et présente une morphologie particulière qui a changé au cours de ces dernières années, d'une forme individualisée à une forme rattachée au cordon littoral de l'estran. Les facteurs à l'origine de l'évolution de cette flèche constitueront l'objectif de cette étude.

$$
\text { DOI: } 10.5150 / \mathrm{cmcm} .2009 .022
$$




\section{Matériel et méthode}

Pour mieux cerner les processus à l'origine de cette évolution, et comprendre la morphodynamique de cette frange littorale, on s'est appuyé sur deux approches. La première approche, analytique, est basée sur l'étude des images satellitaires récentes (Google Earth, 2007) et des photographies aériennes d'une mission de 1997, complétée par des observations sur le terrain et par une étude sédimentologique. La deuxième approche, numérique, est basée sur l'étude hydrodynamique par le biais du système de modélisation côtière (S.M.C.). La cartographie du trait de côte a été réalisée au sein de l'Office Tunisien de la Topographie et Cartographie (O.T.C.) par la restitution photogrammétrique qui nous permet d'obtenir le trait de côte géoréférencé et corrigé. Le trait de côte est assimilé à la ligne de rivage de pleine mer, facilement identifiable sur les photographies aériennes, par la différence radiométrique entre les parties sèches et humides de l'estran (DOLAN et al., 1978 ; DOUGLAS \& CROWELL, 2000).

\section{Evolution de la flèche littorale depuis 1997 jusqu'à 2007}

Durant la période qui précède 1997, le littoral a été largement alimenté surtout pendant les périodes de crues comme celles de 1984, 1990 et 1995 où les précipitations ont été respectivement $370 \mathrm{~mm}, 380 \mathrm{~mm}$ et $550 \mathrm{~mm}$ (D.R.E., 2002). Les pluies dans la région de Gabès sont torrentielles, et donc à l'origine d'importants apports de sédiments fluviatiles jusqu'à la côte. En conséquence, un ensablement important apparaît sur les photographies aériennes de 1997, le long du littoral sud de l'oued Sourrag. Une flèche littorale s'est construite, une première partie rattachée sur le continent au cordon littoral, suivie d'une deuxième partie délimitée par deux chenaux de marée (Fig. 1). Ces chenaux alimentent et drainent la lagune abritée par la flèche : le chenal nord coupe la flèche dans sa partie amont-dérive, le chenal sud en limite la progression vers le SE. Sur l'image satellitaire Google Earth de 2007, cette configuration géomorphologique a été modifiée. En effet, la superposition du trait de côte de 1997 sur cette image montre que la flèche isolée s'est rattachée au cordon sableux amont-dérive et le chenal nord a disparu, alors que le chenal à l'extrémité de la flèche est toujours actif. De plus, la flèche s'est allongée vers le SE pour atteindre plus de 4 km de long (Fig. 1).

\section{Discussion}

L'évolution de la flèche littorale depuis 1997 jusqu'à 2007, a montré que le littoral Sud de la ville de Gabès, est assujetti à une dynamique sédimentaire active. Au niveau de ce littoral se jette l'oued Sourrag, dont le large bassin versant de superficie $340 \mathrm{~km}^{2}$ est drainé violemment lors des épisodes de fortes précipitations, provoquant des crues très chargées en sédiments, comme les crues anciennes de 1973 (volume sédimentaire transporté de $5616 \times 10^{3} \mathrm{~m}^{3}$; BOURGES, 1974), ou les plus récentes de 2003 et 2005. Ces sédiments fluviatiles participent à l'engraissement des côtes car, à l'arrivée dans la zone d'embouchure, ils entrent dans une hydrodynamique complexe avec l'interaction 
des courants de marée, actifs dans les chenaux d'embouchure, et des courants littoraux (courants de dérives et courants sagittaux). Les courants de dérive littorale sont mis en évidence par le système de modélisation côtière, et sont à l'origine de la formation et l'évolution des flèches qui s'allongent dans le sens de la dérive selon la direction des houles. En effet, les houles de directions $\mathrm{N}$ et NNE, produisent un courant de dérive littorale de direction NW-SE; alors que les houles de directions E, ESE et SE, produisent une dérive littorale de direction SE-NW (BARDI et al., 2009). La progression vers le SE de la flèche étudiée prouve que la dérive littorale qui contrôle son évolution est de direction NW-SE, et donc que les houles dominantes dans cette région sont de direction $\mathrm{N}$ et/ou NNE.

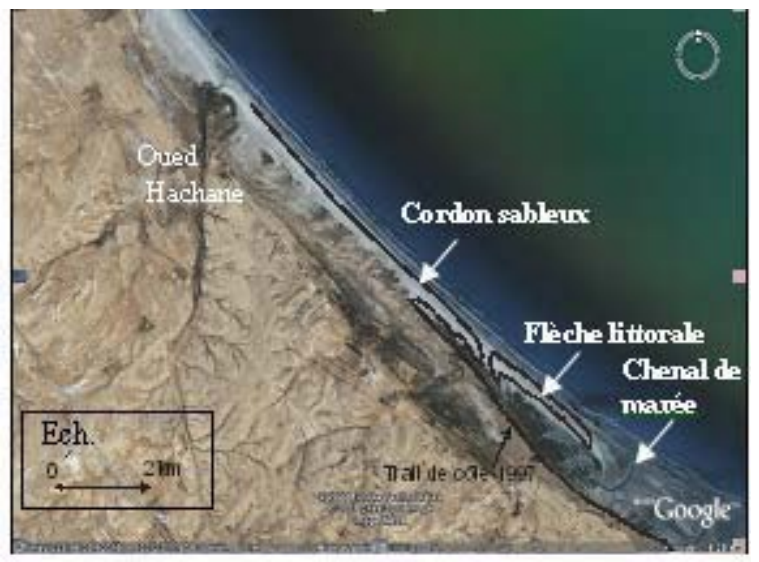

Figure 1. Trait de côte cartographié à partir des photos aériennes de la mission 1997 (trait noir), comparé avec une image Google Earth de 2007 (entre 1997 et 2007 un chenal qui séparait la flèche littoral du cordon sableux a disparu).

La flèche, au sud de l'oued Hachane, qui était séparée du continent par un chenal de marée en 1997, s'y est rattachée et le chenal a été comblé (Fig. 1). Cette évolution morphologique pourrait être attribuée aux tempêtes qui jouent un rôle essentiel dans l'évolution des littoraux (SUANEZ, 1997 ; BRUZZI, 1998 ; DURAND, 1999). Un seul évènement de forte tempête peut remanier assez de sédiments côtiers pour combler un chenal d'embouchure ou le détourner.

Toutefois, un chenal peut se combler aussi par perte de compétence des courants de marée, par diminution du prisme tidal dû par exemple au comblement de la lagune.

De 1997 à 2007, la flèche reste limitée à son extrémité sud par un chenal de marée, de plus de 1,5 m de profondeur, qui bloque sa croissance vers le Sud. Il s'agit donc d'une flèche contrainte (KRAUS, 1999). D'après BALOUIN (2001), les courants tidaux canalisés au niveau des chenaux, puissants surtout pendant les périodes de vives eaux, constituent une véritable barrière hydrodynamique au transit littoral. Ces courants sont capables d'évacuer vers le large le matériel sableux amené dans le chenal par la dérive littorale, et ils érodent ainsi le sédiment accumulé à l'extrémité de la flèche et 
ralentissant ou stoppant sa croissance (KRAUS, 1999). La persistance du chenal Sud de la flèche étudié, prouve que les processus de remplissage du chenal par le transport littoral et son érosion par les courants tidaux maintiennent cette zone en équilibre dynamique (ESCOFFIER, 1940).

\section{Conclusion}

La formation et l'évolution de la flèche littorale au Sud de l'oued Sourrag, est contrôlée d'une part par les apports continentaux en provenance de l'oued lors des crues, qui alimentent la côte en matériel détritique, et d'autre part par les courants littoraux qui les redistribuent le long de la côte. Les chenaux de marée jouent un rôle important dans la croissance de cette flèche puisqu'ils la contraignent et la maintiennent en équilibre dynamique lorsqu'ils se développent au niveau de son extrémité.

\section{Références bibliographiques}

BARDI I. (2002). Etude environnementale de l'estuaire de l'Oued el Ferd (Golfe de Gabès). Caractérisation sédimentologique, géomorphologique et géochimique. DEA, Université Tunis El Manar, $121 \mathrm{p}$.

BARDI I. SOUAYED M., ABDELJAOUAD S., GHARBI W., SOUSSI R. (2009). Hydrodynamique Sédimentaire du littorale Sud de la ville de Gabès (Golfe de Gabès - Sud Tunisien). Revue Geo-Eco-Trop $\mathrm{n}^{\circ} 33$ sous presse.

BALOUIN Y. (2001). Les embouchures mésotidales (tidal inlets) et leur relation avec les littoraux adjacents - Exemple de la Barra Nova, Sud Portugal. Thèse de doctorat geologie marine océanographie, Université Bordeaux I, 302 p.

BEN OUEZDOU H. (1987). Etude morphologique et stratigraphique des formations quaternaires des alentours du Golfe de Gabès. Revue des Sciences de la Terre. INRST, Tunisie. Vol. 5, 165 p.

BOURGES J. (1974). Aperçu sur l'hydrologie du centre Sud tunisien. Réseau d'observation et crues exceptionnelles. Rapp. Int. D.R.E.

BRUZZI C. (1998). Les tempêtes et l'évolution morphosédimentaire des plages orientales du delta du Rhône. Thèse de doctorat, Université de Provence, 325 p.

D.R.E. -Direction de Ressource en Eau- (2002). Rapport interne. Gabès, Tunisie.

DOLAN R., HAYDEN B., HEYWOOD J. (1978). A new photogrammetric method for determining shoreline erosion. Coastal engineering, 2, pp 21-39.

DOUGLAS B.C., CROWELL M. (2000). Long term shoreline prediction and error propagation. Journal of Coastal Research, 16, 1, pp 145-152.

DURAND P. (1999). L'évolution des plages de l'ouest du golfe du Lion au XXème siècle. Cinématique du trait de côte, dynamique sédimentaire, analyse prévisionnelle. Thèse de doctorat, $461 \mathrm{p}$.

ESCOFFIER F.F. (1940). The stability of tidal inlets. Shore and Beach, 8, pp 114-115.

KRAUS N.C. (1999). Analytical model of spit evolution at inlets. Proceedings of Coastal Sediments 99, ASCE, 1999, New York, Long Island, pp 1739-1754.

MAGHREBI S. (1995). Dynamique sédimentaire dans le Golfe de Gabès -Tunisie-, Impact des aménagements côtiers. Thèse Université de Paris Sud, 174 p.

SUANEZ S. (1997). Dynamique sédimentaires actuelles et récentes de la frange littorale orientale du delta du Rhône. Thèse de doctorat, Université de Provence, 283 p. 